\title{
A constructive way to design a switching rule and switching regions to mean square exponential stability of switched stochastic systems with non-differentiable and interval time-varying delay
}

Manlika Rajchakit ${ }^{1}$, Piyapong Niamsup ${ }^{2,3}$ and Grienggrai Rajchakit ${ }^{*}$

${ }^{*}$ Correspondence: griengkrai@yahoo.com

1 Division of Mathematics and

Statistics, Faculty of Science, Maejo

University, Chiangmai, 50290

Thailand

Full list of author information is

available at the end of the article

\section{空 Springer}

\begin{abstract}
This paper addresses a mean square exponential stability problem for a class of switched stochastic systems with time-varying delay. The time delay is any continuous function belonging to a given interval, but not necessary differentiable. By constructing a suitable augmented Lyapunov-Krasovskii functional combined with Leibniz-Newton's formula, new delay-dependent sufficient conditions for the mean square exponential stability of switched stochastic systems with time-varying delay are first established in terms of LMIs. Numerical example is given to show the effectiveness of the obtained result.

MSC: 15A09; 52A10; 74M05; 93D05

Keywords: switching design; mean square exponential stability; switched stochastic systems; scalar Wiener process; Brownian motion; interval delay; Lyapunov function; linear matrix inequalities
\end{abstract}

\section{Introduction}

In the past decades, the problem of stability for neutral differential systems, which have delays in both their state and the derivatives of their states, has been widely investigated by many researchers. Such systems are often encountered in engineering, biology, and economics. The existence of time delay is frequently a source of instability or poor performance in the systems. Recently, some stability criteria for a neutral system with time delay have been given [1-25]. Stability analysis of linear systems with time-varying delays $\dot{x}(t)=A x(t)+D x(t-h(t))$ is fundamental to many practical problems and has received considerable attention [1-7]. In [8-17], which are not based on the method of Lyapunov functional, one of them uses the diagonal equations for reducing systems of delay differential equations to ones of integral equations and estimates the norms or spectral radii of corresponding integral operators obtained on the basis of the results in the book. Most of the known results on this problem are derived assuming only that the time-varying delay $h(t)$ is a continuously differentiable function, satisfying some boundedness condition on its derivative: $\dot{h}(t) \leq \delta<1$. In delay-dependent stability criteria, the main concern is to enlarge the feasible region of stability criteria in a given time-delay interval. Interval

(C2013 Rajchakit et al.: licensee Springer. This is an Open Access article distributed under the terms of the Creative Commons Attribution License (http://creativecommons.org/licenses/by/2.0), which permits unrestricted use, distribution, and reproduction in any medium, provided the original work is properly cited. 
time-varying delay means that a time delay varies in an interval in which the lower bound is not restricted to be zero. By constructing a suitable argument, Lyapunov functional and utilizing free weight matrices, some less conservative conditions for asymptotic stability are derived in [18-24] for systems with time delay varying in an interval. However, the shortcoming of the method used in these works is that the delay function is assumed to be differential and its derivative is still bounded: $\dot{h}(t) \leq \delta$. To the best of our knowledge, a constructive way to design a switching rule, switching regions, and mean square exponential stability of switched stochastic systems with interval time-varying delay, nondifferentiable time-varying delays, which are important in both theory and applications, have not been fully studied yet (see, e.g., [25-38] and the references therein). This motivates our research.

This paper gives the improved results for the mean square exponential stability of switched stochastic systems with interval time-varying delay. The time delay is assumed to be a time-varying continuous function belonging to a given interval, but not necessary differentiable. Specifically, our goal is to develop a constructive way to design a switching rule to exponential stability of switched stochastic systems with interval time-varying delay. By constructing a Lyapunov functional combined with the LMI technique, we propose new criteria for the mean square exponential stability of switched stochastic systems with interval time-varying delay. The delay-dependent mean square exponential stability conditions are formulated in terms of LMIs, being thus solvable by utilizing Matlab's LMI control toolbox available in the literature to date.

The paper is organized as follows. Section 2 presents definitions and some well-known technical propositions needed for the proof of the main results. Delay-dependent mean square exponential stability conditions of switched stochastic systems with interval timevarying delay are presented in Section 3. Numerical example is provided to illustrate the theoretical results in Section 4, and the conclusions are drawn in Section 5.

\section{Preliminaries}

The following notations will be used in this paper. $R^{+}$denotes the set of all real nonnegative numbers; $R^{n}$ denotes the $n$-dimensional space with the scalar product $\langle\cdot, \cdot\rangle$ and the vector norm $\|\cdot\| ; M^{n \times r}$ denotes the space of all matrices of $(n \times r)$-dimensions; $A^{T}$ denotes the transpose of matrix $A$; $A$ is symmetric if $A=A^{T}$; I denotes the identity matrix; $\lambda(A)$ denotes the set of all eigenvalues of $A ; \lambda_{\min / \max }(A)=\min / \max \{\operatorname{Re} \lambda ; \lambda \in \lambda(A)\}$; $x_{t}:=\{x(t+s): s \in[-h, 0]\},\left\|x_{t}\right\|=\sup _{s \in[-h, 0]}\|x(t+s)\| ; C\left([0, t], R^{n}\right)$ denotes the set of all $R^{n}$-valued continuous functions on [0,t]; matrix $A$ is called semi-positive definite $(A \geq 0)$ if $\langle A x, x\rangle \geq 0$ for all $x \in R^{n} ; A$ is positive definite $(A>0)$ if $\langle A x, x\rangle>0$ for all $x \neq 0 ; A>B$ means $A-B>0 . *$ denotes the symmetric term in a matrix.

Consider a switched stochastic system with interval time-varying delay of the form

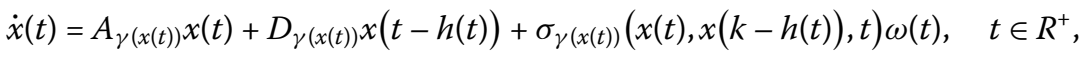

$$
\begin{aligned}
& x(t)=\phi(t), \quad t \in\left[-h_{2}, 0\right] \text {, }
\end{aligned}
$$

where $x(t) \in R^{n}$ is the state; $\gamma(\cdot): R^{n} \rightarrow \mathcal{N}:=\{1,2, \ldots, N\}$ is the switching rule, which is a function depending on the state at each time and will be designed. A switching function is a rule which determines a switching sequence for a given switching system. Moreover, 
$\gamma(x(t))=i$ implies that the system realization is chosen as the $i$ th system, $i=1,2, \ldots, N$. It is seen that system (2.1) can be viewed as an autonomous switched system in which the effective subsystem changes when the state $x(t)$ hits predefined boundaries. $A_{i}, D_{i} \in M^{n \times n}$, $i=1,2, \ldots, N$, are given constant matrices, and $\phi(t) \in C\left(\left[-h_{2}, 0\right], R^{n}\right)$ is the initial function with the norm $\|\phi\|=\sup _{s \in\left[-h_{2}, 0\right]}\|\phi(s)\|$.

$\omega(k)$ is a scalar Wiener process (Brownian motion) on $(\Omega, \mathcal{F}, \mathcal{P})$ with

$$
E\{\omega(t)\}=0, \quad E\left\{\omega^{2}(t)\right\}=1, \quad E\{\omega(i) \omega(j)\}=0 \quad(i \neq j)
$$

and $\sigma_{i}: R^{n} \times R^{n} \times R \rightarrow R^{n}, i=1,2, \ldots, N$, is the continuous function, and it is assumed to satisfy that

$$
\begin{aligned}
& \sigma_{i}^{T}(x(t), x(t-h(t)), t) \sigma_{i}(x(t), x(t-h(t)), t) \\
& \leq \rho_{i 1} x^{T}(t) x(t)+\rho_{i 2} x^{T}(t-h(t)) x(t-h(t)), \\
& \quad x(t), x(t-h(t)) \in R^{n},
\end{aligned}
$$

where $\rho_{i 1}>0$ and $\rho_{i 2}>0, i=1,2, \ldots, N$, are known constant scalars. For simplicity, we denote $\sigma_{i}(x(t), x(t-h(t)), t)$ by $\sigma_{i}$, respectively.

The time-varying delay function $h(t)$ satisfies

$$
0 \leq h_{1} \leq h(t) \leq h_{2}, \quad t \in R^{+} .
$$

The mean square stability problem for switched stochastic system (2.1) is to construct a switching rule that makes the system mean square exponentially stable.

Definition 2.1 Given $\alpha>0$. Switched stochastic system (2.1) is $\alpha$-exponentially stable in the mean square if there exists a switching rule $\gamma(\cdot)$ such that every solution $x(t, \phi)$ of the system satisfies the following condition:

$$
\exists N>0: E\{\|x(t, \phi)\|\} \leq E\left\{N e^{-\alpha t}\|\phi\|\right\}, \quad \forall t \in R^{+} .
$$

Definition 2.2 The system of matrices $\left\{J_{i}\right\}, i=1,2, \ldots, N$, is said to be strictly complete if for every $x \in R^{n} \backslash\{0\}$, there is $i \in\{1,2, \ldots, N\}$ such that $x^{T} J_{i} x<0$.

It is easy to see that the system $\left\{J_{i}\right\}$ is strictly complete if and only if

$$
\bigcup_{i=1}^{N} \alpha_{i}=R^{n} \backslash\{0\}
$$

where

$$
\alpha_{i}=\left\{x \in R^{n}: x^{T} J_{i} x<0\right\}, \quad i=1,2, \ldots, N .
$$

We end this section with the following technical well-known propositions, which will be used in the proof of the main results. 
Proposition 2.1 [39] The system $\left\{J_{i}\right\}, i=1,2, \ldots, N$, is strictly complete if there exist $\delta_{i} \geq 0$, $i=1,2, \ldots, N, \sum_{i=1}^{N} \delta_{i}>0$ such that

$$
\sum_{i=1}^{N} \delta_{i} J_{i}<0
$$

If $N=2$, then the above condition is also necessary for the strict completeness.

Proposition 2.2 (Cauchy inequality) For any symmetric positive definite matrix $N \in$ $M^{n \times n}$ and $a, b \in R^{n}$, we have

$$
\pm a^{T} b \leq a^{T} N a+b^{T} N^{-1} b .
$$

Proposition 2.3 [40] For any symmetric positive definite matrix $M \in M^{n \times n}$, scalar $\mu>0$ and vector function $\omega:[0, \mu] \rightarrow R^{n}$ such that the integrations concerned are well defined, the following inequality holds:

$$
\left(\int_{0}^{\mu} \omega(s) d s\right)^{T} M\left(\int_{0}^{\mu} \omega(s) d s\right) \leq \mu\left(\int_{0}^{\mu} \omega^{T}(s) M \omega(s) d s\right) .
$$

Proposition 2.4 [41, p.89-90] Let $E, H$ and $F$ be any constant matrices of appropriate dimensions and $F^{T} F \leq I$. For any $\epsilon>0$, we have

$$
E F H+H^{T} F^{T} E^{T} \leq \epsilon E E^{T}+\epsilon^{-1} H^{T} H .
$$

Proposition 2.5 (Schur complement lemma [42]) Given constant matrices $X, Y, Z$ with appropriate dimensions satisfying $X=X^{T}, Y=Y^{T}>0$. Then $X+Z^{T} Y^{-1} Z<0$ if and only if

$$
\left(\begin{array}{cc}
X & Z^{T} \\
Z & -Y
\end{array}\right)<0 \quad \text { or } \quad\left(\begin{array}{cc}
-Y & Z \\
Z^{T} & X
\end{array}\right)<0 .
$$

\section{Main results}

In this section, we investigate the mean square exponential stability problem for a class of switched stochastic systems (2.1) with time-varying delay. Before introducing the main result, the following notations of several matrix variables are defined for simplicity,

$$
\begin{aligned}
\mathcal{M}_{i}= & {\left[\begin{array}{ccccc}
M_{11} & M_{12} & M_{13} & M_{14} & M_{15} \\
* & M_{22} & 0 & M_{24} & M_{25} \\
* & * & M_{33} & M_{34} & M_{35} \\
* & * & * & M_{44} & M_{45} \\
* & * & * & * & M_{55}
\end{array}\right], } \\
M_{11}= & A_{i}^{T} P+P A_{i}+2 \alpha P-e^{-2 \alpha h_{1}} R \\
& -e^{-2 \alpha h_{2}} R+Q+2 \rho_{i 1} I, \\
M_{12}= & e^{-2 \alpha h_{1}} R-S_{2} A_{i}, \\
M_{13}= & e^{-2 \alpha h_{2}} R-S_{3} A_{i},
\end{aligned}
$$




$$
\begin{aligned}
& M_{34}=e^{-2 \alpha h_{2}} U-S_{3} D_{i}, \\
& M_{35}=S_{3}, \\
& M_{44}=-2 S_{4} D_{i}-2 e^{-2 \alpha h_{2}} U+2 \rho_{i 2} I, \\
& M_{45}=S_{4}-S_{5} D_{i}, \\
& M_{55}=S_{5}+S_{5}^{T}+h_{1}^{2} R+h_{2}^{2} R+\left(h_{2}-h_{1}\right)^{2} U, \\
& J_{i}=Q-S_{1} A_{i}-A_{i}^{T} S_{1}^{T}, \\
& \alpha_{i}=\left\{x \in R^{n}: x^{T} J_{i} x<0\right\}, \quad i=1,2, \ldots, N, \\
& \bar{\alpha}_{1}=\alpha_{1}, \quad \bar{\alpha}_{i}=\alpha_{i} \backslash \bigcup_{j=1}^{i-1} \bar{\alpha}_{j}, \quad i=2,3, \ldots, N, \\
& \lambda_{1}=\lambda_{\min }(P), \\
& \lambda_{2}=\lambda_{\max }(P)+2 h_{2} \lambda_{\max }(Q)+2 h_{2}^{2} \lambda_{\max }(R) \\
& \quad+\left(h_{2}-h_{1}\right)^{2} \lambda_{\max }(U) .
\end{aligned}
$$

The following is the main result of the paper, which gives sufficient conditions for mean square exponential stability problem for a class of switched stochastic systems (2.1) with time-varying delay.

Theorem 3.1 Given $\alpha>0$. The zero solution of switched stochastic system (2.1) is $\alpha$ exponentially stable in the mean square if there exist symmetric positive definite matrices $P, Q, R, U$, and matrices $S_{i}, i=1,2, \ldots, 5$, satisfying the following conditions:

(i) $\exists \delta_{i} \geq 0, i=1,2, \ldots, N, \sum_{i=1}^{N} \delta_{i}>0: \sum_{i=1}^{N} \delta_{i} J_{i}<0$,

(ii) $\mathcal{M}_{i}<0, i=1,2, \ldots, N$.

The switching rule is chosen as $\gamma(x(t))=i$, whenever $x(t) \in \bar{\alpha}_{i}$. Moreover, the solution $x(t, \phi)$ of the switched stochastic system satisfies

$$
E\{\|x(t, \phi)\|\} \leq E\left\{\sqrt{\frac{\lambda_{2}}{\lambda_{1}}} e^{-\alpha t}\|\phi\|\right\}, \quad \forall t \in R^{+}
$$

Proof We consider the following Lyapunov-Krasovskii functional for system (2.1):

$$
E\left\{V\left(t, x_{t}\right)\right\}=E\left\{\sum_{i=1}^{6} V_{i}\right\},
$$


where

$$
\begin{aligned}
& V_{1}=x^{T}(t) P x(t), \\
& V_{2}=\int_{t-h_{1}}^{t} e^{2 \alpha(s-t)} x^{T}(s) Q x(s) d s, \\
& V_{3}=\int_{t-h_{2}}^{t} e^{2 \alpha(s-t)} x^{T}(s) Q x(s) d s, \\
& V_{4}=h_{1} \int_{-h_{1}}^{0} \int_{t+s}^{t} e^{2 \alpha(\tau-t)} \dot{x}^{T}(\tau) R \dot{x}(\tau) d \tau d s, \\
& V_{5}=h_{2} \int_{-h_{2}}^{0} \int_{t+s}^{t} e^{2 \alpha(\tau-t)} \dot{x}^{T}(\tau) R \dot{x}(\tau) d \tau d s, \\
& V_{6}=\left(h_{2}-h_{1}\right) \int_{t-h_{2}}^{t-h_{1}} \int_{t+s}^{t} e^{2 \alpha(\tau-t)} \dot{x}^{T}(\tau) U \dot{x}(\tau) d \tau d s .
\end{aligned}
$$

It easy to check that

$$
E\left\{\lambda_{1}\|x(t)\|^{2}\right\} \leq E\left\{V\left(t, x_{t}\right)\right\} \leq E\left\{\lambda_{2}\left\|x_{t}\right\|^{2}\right\}, \quad \forall t \geq 0 .
$$

Taking the derivative of $V_{1}$ along the solution of system (2.1) and taking the mathematical expectation, we obtain

$$
\begin{aligned}
E\left\{\dot{V}_{1}\right\} & =E\left\{2 x^{T}(t) P \dot{x}(t)\right\} \\
& =E\left\{x^{T}(t)\left[A_{i}^{T} P+A_{i} P\right] x(t)+2 x^{T}(t) P D_{i} x(t-h(t))+2 x^{T}(t) P \sigma_{i} \omega(t)\right\} ; \\
E\left\{\dot{V}_{2}\right\} & =E\left\{x^{T}(t) Q x(t)-e^{-2 \alpha h_{1}} x^{T}\left(t-h_{1}\right) Q x\left(t-h_{1}\right)-2 \alpha V_{2}\right\} ; \\
E\left\{\dot{V}_{3}\right\} & =E\left\{x^{T}(t) Q x(t)-e^{-2 \alpha h_{2}} x^{T}\left(t-h_{2}\right) Q x\left(t-h_{2}\right)-2 \alpha V_{3}\right\} ; \\
E\left\{\dot{V}_{4}\right\} & =E\left\{h_{1}^{2} \dot{x}^{T}(t) R \dot{x}(t)-h_{1} \int_{t-h_{1}}^{t} e^{2 \alpha(\tau-t)} \dot{x}^{T}(s) R \dot{x}(s) d s-2 \alpha V_{4}\right\} \\
& \leq E\left\{h_{1}^{2} \dot{x}^{T}(t) R \dot{x}(t)-h_{1} e^{-2 \alpha h_{1}} \int_{t-h_{1}}^{t} \dot{x}^{T}(s) R \dot{x}(s) d s-2 \alpha V_{4}\right\} ; \\
E\left\{\dot{V}_{5}\right\} & =E\left\{h_{2}^{2} \dot{x}^{T}(t) R \dot{x}(t)-h_{2} \int_{t-h_{2}}^{t} e^{2 \alpha(\tau-t)} \dot{x}^{T}(s) R \dot{x}(s) d s-2 \alpha V_{5}\right\} \\
& \leq E\left\{h_{2}^{2} \dot{x}^{T}(t) R \dot{x}(t)-h_{2} e^{-2 \alpha h_{2}} \int_{t-h_{2}}^{t} \dot{x}^{T}(s) R \dot{x}(s) d s-2 \alpha V_{5}\right\} ; \\
E\left\{\dot{V}_{6}\right\} & \leq E\left\{\left(h_{2}-h_{1}\right)^{2} \dot{x}^{T}(t) U \dot{x}(t)-\left(h_{2}-h_{1}\right) e^{-2 \alpha h_{2}} \int_{t-h_{2}}^{t-h_{1}} \dot{x}^{T}(s) U \dot{x}(s) d s-2 \alpha V_{6}\right\} .
\end{aligned}
$$

Applying Proposition 2.2 and the Leibniz-Newton formula, we have

$$
\begin{aligned}
E\left\{-h_{i} \int_{t-h_{i}}^{t} \dot{x}^{T}(s) R \dot{x}(s) d s\right\} & \leq E\left\{-\left[\int_{t-h_{i}}^{t} \dot{x}(s) d s\right]^{T} R\left[\int_{t-h_{i}}^{t} \dot{x}(s) d s\right]\right\} \\
& \leq E\left\{-\left[x(t)-x\left(t-h_{i}\right)\right]^{T} R\left[x(t)-x\left(t-h_{i}\right)\right]\right\} \\
& =E\left\{-x^{T}(t) R x(t)+2 x^{T}(t) R x\left(t-h_{i}\right)-x^{T}\left(t-h_{i}\right) R x\left(t-h_{i}\right)\right\} .
\end{aligned}
$$


Note that

$$
E\left\{\int_{t-h_{2}}^{t-h_{1}} \dot{x}^{T}(s) U \dot{x}(s) d s\right\}=E\left\{\int_{t-h_{2}}^{t-h(t)} \dot{x}^{T}(s) U \dot{x}(s) d s+\int_{t-h(t)}^{t-h_{1}} \dot{x}^{T}(s) U \dot{x}(s) d s\right\} .
$$

Using Proposition 2.2 gives

$$
\begin{aligned}
& E\left\{\left[h_{2}-h(t)\right] \int_{t-h_{2}}^{t-h(t)} \dot{x}^{T}(s) U \dot{x}(s) d s\right\} \\
& \quad \geq E\left\{\left[\int_{t-h_{2}}^{t-h(t)} \dot{x}(s) d s\right]^{T} U\left[\int_{t-h_{2}}^{t-h(t)} \dot{x}(s) d s\right]\right\} \\
& \quad \geq E\left\{\left[x(t-h(t))-x\left(t-h_{2}\right)\right]^{T} U\left[x(t-h(t))-x\left(t-h_{2}\right)\right]\right\} .
\end{aligned}
$$

Since $h_{2}-h(t) \leq h_{2}-h_{1}$, we have

$$
\begin{aligned}
& E\left\{\left[h_{2}-h_{1}\right] \int_{t-h_{2}}^{t-h(t)} \dot{x}^{T}(s) U \dot{x}(s) d s\right\} \\
& \quad \geq E\left\{\left[x(t-h(t))-x\left(t-h_{2}\right)\right]^{T} U\left[x(t-h(t))-x\left(t-h_{2}\right)\right]\right\},
\end{aligned}
$$

then

$$
\begin{aligned}
& E\left\{-\left(h_{2}-h_{1}\right) \int_{t-h_{2}}^{t-h(t)} \dot{x}^{T}(s) U \dot{x}(s) d s\right\} \\
& \quad \leq E\left\{-\left[x(t-h(t))-x\left(t-h_{2}\right)\right]^{T} U\left[x(t-h(t))-x\left(t-h_{2}\right)\right]\right\} .
\end{aligned}
$$

Similarly, we have

$$
\begin{aligned}
& E\left\{-\left(h_{2}-h_{1}\right) \int_{t-h(t)}^{t-h_{1}} \dot{x}^{T}(s) U \dot{x}(s) d s\right\} \\
& \quad \leq E\left\{-\left[x\left(t-h_{1}\right)-x(t-h(t))\right]^{T} U\left[x\left(t-h_{1}\right)-x(t-h(t))\right]\right\} .
\end{aligned}
$$

Therefore, we have

$$
\begin{aligned}
E\{\dot{V}(\cdot) & +2 \alpha V(\cdot)\} \\
\leq & E\left\{x^{T}(t)\left[A_{i}^{T} P+A_{i} P+2 \alpha P+2 Q\right] x(t)\right\} \\
& +E\left\{2 x^{T}(t) P D_{i} x(t-h(t))+2 x^{T}(t) P \sigma_{i} \omega(t)\right\} \\
& +E\left\{-e^{-2 \alpha h_{1}} x^{T}\left(t-h_{1}\right) Q x\left(t-h_{1}\right)\right\} \\
& +E\left\{-e^{-2 \alpha h_{2}} x^{T}\left(t-h_{2}\right) Q x\left(t-h_{2}\right)\right\} \\
& +E\left\{\dot{x}^{T}(t)\left[\left(h_{1}^{2}+h_{2}^{2}\right) R+\left(h_{2}-h_{1}\right)^{2} U\right] \dot{x}(t)\right\} \\
& +E\left\{-e^{-2 \alpha h_{1}}\left[x(t)-x\left(t-h_{1}\right)\right]^{T} R\left[x(t)-x\left(t-h_{1}\right)\right]\right\} \\
& +E\left\{-e^{-2 \alpha h_{2}}\left[x(t)-x\left(t-h_{2}\right)\right]^{T} R\left[x(t)-x\left(t-h_{2}\right)\right]\right\} \\
& +E\left\{-e^{-2 \alpha h_{2}}\left[x(t-h(t))-x\left(t-h_{2}\right)\right]^{T} U\left[x(t-h(t))-x\left(t-h_{2}\right)\right]\right\} \\
& +E\left\{-e^{-2 \alpha h_{2}}\left[x\left(t-h_{1}\right)-x(t-h(t))\right]^{T} U\left[x\left(t-h_{1}\right)-x(t-h(t))\right]\right\} .
\end{aligned}
$$


By using the following identity relation

$$
\dot{x}(t)-A_{i} x(t)-D_{i} x(t-h(t))=0,
$$

and multiplying by $2 x^{T}(t) S_{1}, 2 x^{T}\left(t-h_{1}\right) S_{2}, 2 x^{T}\left(t-h_{2}\right) S_{3}, 2 x^{T}(t-h(t)) S_{4}, 2 \dot{x}^{T}(t) S_{5}$, $2 \omega^{T}(t) \sigma_{i}^{T}$ both sides of the identity relation, we have

$$
\begin{aligned}
& 2 x^{T}(t) S_{1} \dot{x}(t)-2 x^{T}(t) S_{1} A_{i} x(t)-2 x^{T}(t) S_{1} D_{i} x(t-h(t))-2 x^{T}(t) S_{1} \sigma_{i} \omega(t)=0, \\
& 2 x^{T}\left(t-h_{1}\right) S_{2} \dot{x}(t)-2 x^{T}\left(t-h_{1}\right) S_{2} A_{i} x(t) \\
& \quad-2 x^{T}\left(t-h_{1}\right) S_{2} D_{i} x(t-h(t))-2 x^{T}\left(t-h_{1}\right) S_{2} \sigma_{i} \omega(t)=0, \\
& 2 x^{T}\left(t-h_{2}\right) S_{3} \dot{x}(t)-2 x^{T}\left(t-h_{2}\right) S_{3} A_{i} x(t) \\
& \quad-2 x^{T}\left(t-h_{2}\right) S_{3} D_{i} x(t-h(t))-2 x^{T}\left(t-h_{2}\right) S_{3} \sigma_{i} \omega(t)=0, \\
& 2 x^{T}(t-h(t)) S_{4} \dot{x}(t)-2 x^{T}(t-h(t)) S_{4} A_{i} x(t) \\
& \quad-2 x^{T}(t-h(t)) S_{4} D_{i} x(t-h(t))-2 x^{T}(t-h(t)) S_{4} \sigma_{i} \omega(t)=0, \\
& 2 \dot{x}^{T}(t) S_{5} \dot{x}(t)-2 \dot{x}^{T}(t) S_{5} A_{i} x(t)-2 \dot{x}^{T}(t) S_{5} D_{i} x(t-h(t))-2 \dot{x}^{T}(t) S_{5} \sigma_{i} \omega(t)=0, \\
& 2 \omega^{T}(t) \sigma_{i}^{T} \dot{x}(t)-2 \omega^{T}(t) \sigma_{i}^{T} A_{i} x(t)-2 \omega^{T}(t) \sigma_{i}^{T} D_{i} x(t-h(t))-2 \omega^{T}(t) \sigma_{i}^{T} \sigma_{i} \omega(t)=0 .
\end{aligned}
$$

Adding all the zero items of (3.4) into (3.3), we obtain

$$
\begin{aligned}
E\{\dot{V}(\cdot)+2 \alpha V(\cdot)\} \leq & E\left\{x^{T}(t)\left[A_{i}^{T} P+P A_{i}+2 \alpha P-e^{-2 \alpha h_{1}} R\right] x(t)\right\} \\
& +E\left\{x^{T}(t)\left[-e^{-2 \alpha h_{2}} R+S_{1} A_{i}+A_{i}^{T} S_{1}^{T}+2 Q\right] x(t)\right\} \\
& +E\left\{2 x^{T}(t)\left[e^{-2 \alpha h_{1}} R-S_{2} A_{i}\right] x\left(t-h_{1}\right)\right\} \\
& +E\left\{2 x^{T}(t)\left[e^{-2 \alpha h_{2}} R-S_{3} A_{i}\right] x\left(t-h_{2}\right)\right\} \\
& +E\left\{2 x^{T}(t)\left[P D_{i}-S_{1} D_{i}-S_{4} A_{i}\right] x(t-h(t))\right\} \\
& +E\left\{2 x^{T}(t)\left[S_{1}-S_{5} A_{i}\right] \dot{x}(t)\right\} \\
& +E\left\{2 x^{T}(t)\left[P \sigma_{i}-S_{1} \sigma_{i}-A_{i}^{T} \sigma_{i}\right] \omega(t)\right\} \\
& +E\left\{x^{T}\left(t-h_{1}\right)\left[-e^{-2 \alpha h_{1}} Q-e^{-2 \alpha h_{1}} R-e^{-2 \alpha h_{2}} U\right] x\left(t-h_{1}\right)\right\} \\
& +E\left\{2 x^{T}\left(t-h_{1}\right)\left[e^{-2 \alpha h_{2}} U-S_{2} D_{i}\right] x(t-h(t))\right\} \\
& +E\left\{2 x^{T}\left(t-h_{1}\right) S_{2} \dot{x}(t)\right\} \\
& +E\left\{2 x^{T}\left(t-h_{1}\right)\left[-S_{2} \sigma_{i}\right] \omega(t)\right\} \\
& +E\left\{x^{T}\left(t-h_{2}\right)\left[-e^{-2 \alpha h_{2}} Q-e^{-2 \alpha h_{2}} R-e^{-2 \alpha h_{2}} U\right] x\left(t-h_{2}\right)\right\} \\
+ & E\left\{2 x^{T}\left(t-h_{2}\right)\left[e^{-2 \alpha h_{2}} U-S_{3} D_{i}\right] x(t-h(t))\right\} \\
+ & E\left\{2 x^{T}\left(t-h_{2}\right) S_{3} \dot{x}(t)\right\} \\
+ & E\left\{2 x^{T}\left(t-h_{2}\right)\left[-S_{3} \sigma_{i}\right] \omega(t)\right\} \\
+ & E\left\{x^{T}(t-h(t))\left[-2 e^{-2 \alpha h_{2}} U-2 S_{4} D_{i}\right] x(t-h(t))\right\} \\
+ & \left\{2 x^{T}(t-h(t))\left[S_{4}-S_{5} D_{i}\right] \dot{x}(t)\right\} \\
& \\
&
\end{aligned}
$$




$$
\begin{aligned}
& +E\left\{2 x^{T}(t-h(t))\left[-S_{4} \sigma_{i}-\sigma_{i}^{T} D_{i}\right] \omega(t)\right\} \\
& +E\left\{\dot{x}^{T}(t)\left[S_{5}+S_{5}^{T}+h_{1}^{2} R+h_{2}^{2} R+\left(h_{2}-h_{1}\right)^{2} U\right] \dot{x}(t)\right\} \\
& +E\left\{2 \dot{x}^{T}(t)\left[\sigma_{i}^{T}-S_{5} \sigma_{i}\right] \omega(t)\right\} \\
& +E\left\{2 \omega^{T}(t)\left[-\sigma_{i}^{T} \sigma_{i}\right] \omega(t)\right\}
\end{aligned}
$$

By assumption (2.2), we have

$$
\begin{aligned}
E\{\dot{V}(\cdot)+2 \alpha V(\cdot)\} \leq & E\left\{x^{T}(t)\left[A_{i}^{T} P+P A_{i}+2 \alpha P-e^{-2 \alpha h_{1}} R\right]\right\} \\
& +E\left\{x^{T}(t)\left[-e^{-2 \alpha h_{2}} R+S_{1} A_{i}+A_{i}^{T} S_{1}^{T}+2 Q\right] x(t)\right\} \\
& +E\left\{2 x^{T}(t)\left[e^{-2 \alpha h_{1}} R-S_{2} A_{i}\right] x\left(t-h_{1}\right)\right\} \\
& +E\left\{2 x^{T}(t)\left[e^{-2 \alpha h_{2}} R-S_{3} A_{i}\right] x\left(t-h_{2}\right)\right\} \\
& +E\left\{2 x^{T}(t)\left[P D_{i}-S_{1} D_{i}-S_{4} A_{i}\right] x(t-h(t))\right\} \\
& +E\left\{2 x^{T}(t)\left[S_{1}-S_{5} A_{i}\right] \dot{x}(t)\right\} \\
& +E\left\{x^{T}\left(t-h_{1}\right)\left[-e^{-2 \alpha h_{1}} Q-e^{-2 \alpha h_{1}} R-e^{-2 \alpha h_{2}} U\right] x\left(t-h_{1}\right)\right\} \\
& +E\left\{2 x^{T}\left(t-h_{1}\right)\left[e^{-2 \alpha h_{2}} U-S_{2} D_{i}\right] x(t-h(t))\right\} \\
& +E\left\{2 x^{T}\left(t-h_{1}\right) S_{2} \dot{x}(t)\right\} \\
& +E\left\{x^{T}\left(t-h_{2}\right)\left[-e^{-2 \alpha h_{2}} Q-e^{-2 \alpha h_{2}} R-e^{-2 \alpha h_{2}} U\right] x\left(t-h_{2}\right)\right\} \\
& +E\left\{x^{T}\left(t-h_{2}\right)\left[e^{-2 \alpha h_{2}} U-S_{3} D_{i}\right] x(t-h(t))\right\} \\
& +E\left\{2 x^{T}\left(t-h_{2}\right) S_{3} \dot{x}(t)\right\} \\
& +E\left\{x^{T}(t-h(t))\left[-2 S_{4} D_{i}-2 e^{-2 \alpha h_{2}} U\right] x(t-h(t))\right\} \\
& +E\left\{2 x^{T}(t-h(t))\left[S_{4}-S_{5} D_{i}\right] \dot{x}(t)\right\} \\
& +E\left\{\dot{x}^{T}(t)\left[S_{5}+S_{5}^{T}+h_{1}^{2} R+h_{2}^{2} R+\left(h_{2}-h_{1}\right)^{2} U\right] \dot{x}(t)\right\} \\
& +E\left\{2\left[-\sigma_{i}^{T} \sigma_{i}\right]\right\} .
\end{aligned}
$$

Applying assumption (2.3), the following estimations hold:

$$
\begin{aligned}
E\{\dot{V}(\cdot)+2 \alpha V(\cdot)\} \leq & E\left\{x^{T}(t)\left[A_{i}^{T} P+P A_{i}+2 \alpha P-e^{-2 \alpha h_{1}} R\right]\right\} \\
& +E\left\{x^{T}(t)\left[-e^{-2 \alpha h_{2}} R+S_{1} A_{i}+A_{i}^{T} S_{1}^{T}+2 Q+2 \rho_{i 1} I\right] x(t)\right\} \\
& +E\left\{2 x^{T}(t)\left[e^{-2 \alpha h_{1}} R-S_{2} A_{i}\right] x\left(t-h_{1}\right)\right\} \\
& +E\left\{2 x^{T}(t)\left[e^{-2 \alpha h_{2}} R-S_{3} A_{i}\right] x\left(t-h_{2}\right)\right\} \\
& +E\left\{2 x^{T}(t)\left[P D_{i}-S_{1} D_{i}-S_{4} A_{i}\right] x(t-h(t))\right\} \\
& +E\left\{2 x^{T}(t)\left[S_{1}-S_{5} A_{i}\right] \dot{x}(t)\right\} \\
& +E\left\{x^{T}\left(t-h_{1}\right)\left[-e^{-2 \alpha h_{1}} Q-e^{-2 \alpha h_{1}} R-e^{-2 \alpha h_{2}} U\right] x\left(t-h_{1}\right)\right\} \\
& +E\left\{2 x^{T}\left(t-h_{1}\right)\left[e^{-2 \alpha h_{2}} U-S_{2} D_{i}\right] x(t-h(t))\right\} \\
& +E\left\{2 x^{T}\left(t-h_{1}\right) S_{2} \dot{x}(t)\right\}
\end{aligned}
$$




$$
\begin{aligned}
& +E\left\{x^{T}\left(t-h_{2}\right)\left[-e^{-2 \alpha h_{2}} Q-e^{-2 \alpha h_{2}} R-e^{-2 \alpha h_{2}} U\right] x\left(t-h_{2}\right)\right\} \\
& +E\left\{x^{T}\left(t-h_{2}\right)\left[e^{-2 \alpha h_{2}} U-S_{3} D_{i}\right] x(t-h(t))\right\} \\
& +E\left\{2 x^{T}\left(t-h_{2}\right) S_{3} \dot{x}(t)\right\} \\
& +E\left\{x^{T}(t-h(t))\left[-2 S_{4} D_{i}-2 e^{-2 \alpha h_{2}} U+2 \rho_{i 2} I\right] x(t-h(t))\right\} \\
& +E\left\{2 x^{T}(t-h(t))\left[S_{4}-S_{5} D_{i}\right] \dot{x}(t)\right\} \\
& +E\left\{\dot{x}^{T}(t)\left[S_{5}+S_{5}^{T}+h_{1}^{2} R+h_{2}^{2} R+\left(h_{2}-h_{1}\right)^{2} U\right] \dot{x}(t)\right\} \\
& =E\left\{x^{T}(t) J_{i} x(t)+\zeta^{T}(t) \mathcal{M}_{i} \zeta(t)\right\}
\end{aligned}
$$

where $\zeta^{T}(t)=\left[x^{T}(t), x^{T}\left(t-h_{1}\right), x^{T}\left(t-h_{2}\right), x^{T}(t-h(t)), \dot{x}^{T}(t)\right]$.

Therefore, we finally obtain from (3.5) and condition (ii) that

$$
E\{\dot{V}(\cdot)+2 \alpha V(\cdot)\}<E\left\{x^{T}(t) J_{i} x(t)\right\}, \quad \forall i=1,2, \ldots, N, t \in R^{+}
$$

We now apply condition (i) and Proposition 2.1, the system $J_{i}$ is strictly complete, and the sets $\alpha_{i}$ and $\bar{\alpha}_{i}$ by (3.1) are well defined such that

$$
\begin{aligned}
& \bigcup_{i=1}^{N} \alpha_{i}=R^{n} \backslash\{0\}, \\
& \bigcup_{i=1}^{N} \bar{\alpha}_{i}=R^{n} \backslash\{0\}, \quad \bar{\alpha}_{i} \cap \bar{\alpha}_{j}=\emptyset, \quad i \neq j .
\end{aligned}
$$

Therefore, for any $x(t) \in R^{n}, t \in R^{+}$, there exists $i \in\{1,2, \ldots, N\}$ such that $x(t) \in \bar{\alpha}_{i}$. By choosing a switching rule as $\gamma(x(t))=i$ whenever $\gamma(x(t)) \in \bar{\alpha}_{i}$, from (3.5) we have

$$
E\{\dot{V}(\cdot)+2 \alpha V(\cdot)\} \leq E\left\{x^{T}(t) J_{i} x(t)\right\}<0, \quad t \in R^{+},
$$

and hence

$$
E\left\{\dot{V}\left(t, x_{t}\right)\right\} \leq E\left\{-2 \alpha V\left(t, x_{t}\right)\right\}, \quad \forall t \in R^{+} .
$$

Integrating both sides of (3.6) from 0 to $t$, we obtain

$$
E\left\{V\left(t, x_{t}\right)\right\} \leq E\left\{V(\phi) e^{-2 \alpha t}\right\}, \quad \forall t \in R^{+} .
$$

Furthermore, taking condition (3.2) into account, we have

$$
E\left\{\lambda_{1}\|x(t, \phi)\|^{2}\right\} \leq E\left\{V\left(x_{t}\right)\right\} \leq E\left\{V(\phi) e^{-2 \alpha t}\right\} \leq E\left\{\lambda_{2} e^{-2 \alpha t}\|\phi\|^{2}\right\},
$$

then

$$
E\{\|x(t, \phi)\|\} \leq E\left\{\sqrt{\frac{\lambda_{2}}{\lambda_{1}}} e^{-\alpha t}\|\phi\|\right\}, \quad t \in R^{+} .
$$


By Definition 2.1, system (2.1) is exponentially stable in the mean square. The proof is complete.

To illustrate the obtained result, let us give the following numerical example.

\section{Numerical example}

Example 4.1 Consider the following switched stochastic systems with interval timevarying delay (2.1), where the delay function $h(t)$ is given by

$$
h(t)=0.2+1.5329 \sin ^{2} t
$$

and

$$
\begin{array}{ll}
A_{1}=\left(\begin{array}{cc}
-2 & 0.1 \\
0.2 & -2.5
\end{array}\right), & A_{2}=\left(\begin{array}{cc}
-2.5 & 0.3 \\
0.2 & -2.9
\end{array}\right), \\
D_{1}=\left(\begin{array}{cc}
-0.3 & 0.2 \\
0.1 & -0.39
\end{array}\right), & D_{2}=\left(\begin{array}{cc}
-0.5 & 0.2 \\
0.1 & -0.4
\end{array}\right) .
\end{array}
$$

It is worth noting that the delay function $h(t)$ is non-differentiable and the exponent $\alpha \geq 1$. Therefore, the methods used in [3, 21, 22, 24-28, 30-39] are not applicable to this system. By LMI toolbox of Matlab, we find that conditions (i), (ii) of Theorem 3.1 are satisfied with $h_{1}=0.1, h_{2}=1.7329, \delta_{1}=0.5, \delta_{2}=0.3, \alpha=1.5, \rho_{11}=0.1, \rho_{12}=0.2, \rho_{21}=0.1, \rho_{22}=0.2$ and

$$
\begin{array}{ll}
P=\left(\begin{array}{cc}
1.2397 & -0.3984 \\
-0.3984 & 1.3112
\end{array}\right), \quad Q=\left(\begin{array}{cc}
1.7931 & -0.0079 \\
-0.0079 & 0.2397
\end{array}\right), \\
R=\left(\begin{array}{cc}
2.3297 & -0.1121 \\
-0.1121 & 1.3397
\end{array}\right), \quad U=\left(\begin{array}{cc}
1.7394 & -0.0982 \\
-0.0982 & 0.6321
\end{array}\right), \\
S_{1}=\left(\begin{array}{cc}
-0.6210 & -0.0335 \\
0.0499 & -0.3576
\end{array}\right), & S_{2}=\left(\begin{array}{cc}
-0.3602 & 0.0170 \\
0.0298 & -0.3550
\end{array}\right), \\
S_{3}=\left(\begin{array}{cc}
-0.3602 & 0.0170 \\
0.0298 & -0.3550
\end{array}\right), & S_{4}=\left(\begin{array}{cc}
0.6968 & -0.0401 \\
-0.0525 & 0.7040
\end{array}\right), \\
S_{5}=\left(\begin{array}{cc}
-1.4043 & 0.0265 \\
-0.0028 & -0.9774
\end{array}\right) . &
\end{array}
$$

In this case, we have

$$
\left(J_{1}, J_{2}\right)=\left(\left[\begin{array}{ll}
-1.5667 & -0.0031 \\
-0.0031 & -1.9712
\end{array}\right],\left[\begin{array}{cc}
-1.5511 & 0.0029 \\
0.0029 & -1.3297
\end{array}\right]\right) \text {. }
$$

Moreover, the sum

$$
\delta_{1} J_{1}(R, Q)+\delta_{2} J_{2}(R, Q)=\left[\begin{array}{cc}
-0.3269 & 0 \\
0 & -0.7239
\end{array}\right]
$$




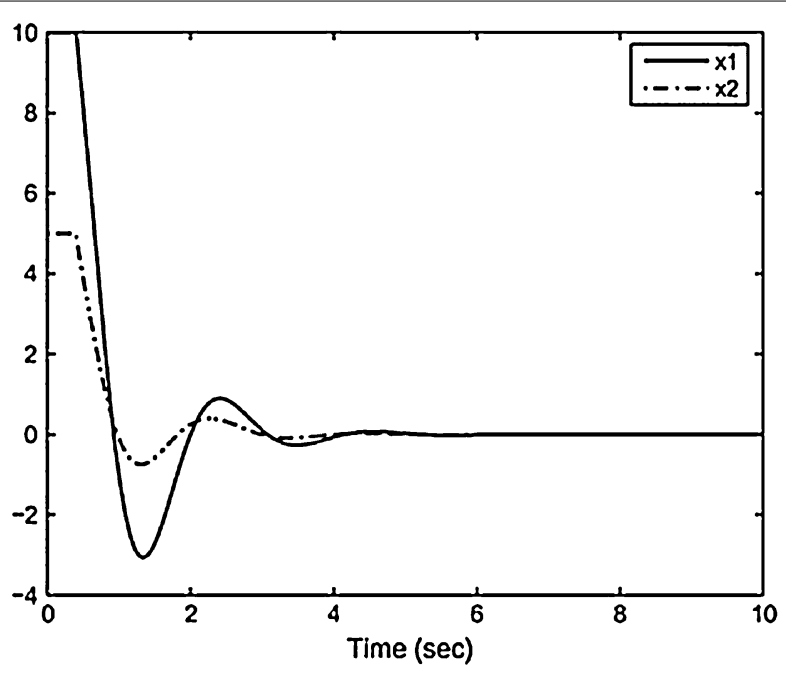

Figure 1 The simulation of the solutions $x_{1}(t)$ and $x_{2}(t)$ with the initial condition $\phi(t)=[105]^{T}$, $t \in[-0.4,0]$.

is negative definite; i.e., the first entry in the first row and the first column $-0.3269<0$ is negative and the determinant of the matrix is positive. The sets $\alpha_{1}$ and $\alpha_{2}$ are given as

$$
\begin{aligned}
& \alpha_{1}=\left\{\left(x_{1}, x_{2}\right):-1.5667 x_{1}^{2}-0.0062 x_{1} x_{2}-1.9712 x_{2}^{2}<0\right\}, \\
& \alpha_{2}=\left\{\left(x_{1}, x_{2}\right): 1.5511 x_{1}^{2}-0.0058 x_{1} x_{2}+1.3297 x_{2}^{2}>0\right\} .
\end{aligned}
$$

Obviously, the union of these sets is equal to $R^{2} \backslash\{0\}$. The switching regions are defined as

$$
\begin{aligned}
& \bar{\alpha}_{1}=\left\{\left(x_{1}, x_{2}\right):-1.5667 x_{1}^{2}-0.0062 x_{1} x_{2}-1.9712 x_{2}^{2}<0\right\} \\
& \bar{\alpha}_{2}=\alpha_{2} \backslash \bar{\alpha}_{1} .
\end{aligned}
$$

By Theorem 3.1, switched stochastic system (2.1) is 1.5-exponentially stable in the mean square and the switching rule is chosen as $\gamma(x(t))=i$ whenever $x(t) \in \bar{\alpha}_{i}$. Moreover, the solution $x(t, \phi)$ of the system satisfies

$$
E\{\|x(t, \phi)\|\} \leq E\left\{1.0239 e^{-1.5 t}\|\phi\|\right\}, \quad \forall t \in R^{+} .
$$

(The trajectories of solution of switched stochastic systems is shown in Figure 1, respectively.)

\section{Conclusions}

In this paper, we have proposed new delay-dependent conditions for the mean square exponential stability of switched stochastic systems with time-varying delay. Based on the improved Lyapunov-Krasovskii functional and the linear matrix inequality technique, a switching rule for the mean square exponential stability of switched stochastic systems with time-varying delay has been established in terms of LMIs. 


\section{Competing interests}

The authors declare that they have no competing interests.

\section{Authors' contributions}

The authors contributed equally and significantly in writing this paper. The authors read and approved the final manuscript.

\section{Author details}

'Division of Mathematics and Statistics, Faculty of Science, Maejo University, Chiangmai, 50290, Thailand. ${ }^{2}$ Department of Mathematics, Faculty of Science, Chiang Mai University, Chiangmai, 50200, Thailand. ${ }^{3}$ Center of Excellence in Mathematics, CHE, Si Ayutthaya Road, Bangkok, 10400, Thailand.

\section{Acknowledgements}

This work was supported by the Thailand Research Fund Grant, the Commission for Higher Education and Faculty of Science, Maejo University, Thailand. The second author is supported by the Center of Excellence in Mathematics, Thailand, and Commission for Higher Education, Thailand. The authors thank anonymous reviewers for valuable comments and suggestions, which allowed us to improve the paper.

Received: 25 March 2013 Accepted: 25 September 2013 Published: 08 Nov 2013

\section{References}

1. de Oliveira, MC, Geromel, JC, Hsu, L: LMI characterization of structural and robust stability: the discrete-time case. Linear Algebra Appl. 296, 27-38 (1999)

2. Phat, VN, Nam, PT: Exponential stability and stabilization of uncertain linear time-varying systems using parameter dependent Lyapunov function. Int. J. Control 80, 1333-1341 (2007)

3. Rajchakit, G: Delay-dependent optimal guaranteed cost control of stochastic neural networks with interval nondifferentiable time-varying delays. Adv. Differ. Equ. 2013, 241 (2013). doi:10.1186/1687-1847-2013-241

4. Sun, YJ: Global stabilizability of uncertain systems with time-varying delays via dynamic observer-based output feedback. Linear Algebra Appl. 353, 91-105 (2002)

5. Phat, VN, Khongtham, Y, Ratchagit, K: LMI approach to exponential stability of linear systems with interval time-varying delays. Linear Algebra Appl. 436, 243-251 (2012)

6. Phat, VN, Ratchagit, K: Stability and stabilization of switched linear discrete-time systems with interval time-varying delay. Nonlinear Anal. Hybrid Syst. 5, 605-612 (2011)

7. Ratchagit, K, Phat, VN: Stability criterion for discrete-time systems. J. Inequal. Appl. 2010, Article ID 201459 (2010)

8. Krasnosel'skii, MA, Vainikko, GM, Zabreiko, PP, Rutitskii, JB, Stezenko, VJ: Approximate Method for Solving Operator Equations. Nauka, Moscow (1969)

9. Azbelev, NV, Maksimov, VP, Rakhmatullina, LF: Introduction to Theory of Linear Functional Differential Equations. Advanced Series in Mathematical Sciences and Engineering, vol. 3. World Federation Publishers Company, Atlanta (1995)

10. Domoshnitsky, A, Sheina, MV: Nonnegativity of Cauchy matrix and stability of systems with delay. Differ. Uravn. 25, 201-208 (1989)

11. Gyori, I: Interaction between oscillation and global asymptotic stability in delay differential equations. Differ. Integral Equ. 3, 181-200 (1990)

12. Gyori, I, Hartung, F: Fundamental solution and asymptotic stability of linear delay differential equations. Dyn. Contin. Discrete Impuls. Syst., Ser. A Math. Anal. 13, 261-287 (2006)

13. Hofbauer, J, So, JW-H: Diagonal dominance and harmless off-diagonal delays. Proc. Am. Math. Soc. 128, 2675-2682 (2000)

14. Campbell, SA: Delay independent stability for additive neural networks. Differ. Equ. Dyn. Syst. 9(3-4), 115-138 (2001)

15. Bainov, D, Domoshnitsky, A: Nonnegativity of the Cauchy matrix and exponential stability of a neutral type system of functional-differential equations. Extr. Math. 8, 75-82 (1993)

16. Domoshnitsky, A: About maximum principles for one of the components of solution vector and stability for systems of linear delay differential equations. Discrete Contin. Dyn. Syst.. 2011, 373-380 (2011). Supplement 2011, Dedicated to the 8th AIMS Conference, Dresden, Germany, American Institute of Mathematical Sciences

17. Gamliel, D, Domoshnitsky, A, Shklyar, R: Time evolution of spin exchange with a time delay. Funct. Differ. Equ. 20, 81-114 (2013)

18. Shatyrko, A, Diblík, J, Khusainov, D, Ruzickova, M: Stabilization of Lur'e-type nonlinear control systems by Lyapunov-Krasovskii functionals. Adv. Differ. Equ. 2012, 229 (2012). doi:10.1186/1687-1847-2012-229

19. Diblík, J, Dzhalladova, I, Ruzickova, M: The stability of nonlinear differential systems with random parameters. Abstr. Appl. Anal. 2012, Article ID 924107 (2012). doi:10.1155/2012/924107

20. Bastinec, J, Diblík, J, Khusainov, DY, Ryvolova, A: Exponential stability and estimation of solutions of linear differential systems of neutral type with constant coefficients. Bound. Value Probl. 2010, Article ID 956121 (2010). doi:10.1155/2010/956121

21. Kwon, OM, Park, JH: Delay-range-dependent stabilization of uncertain dynamic systems with interval time-varying delays. Appl. Math. Comput. 208, 58-68 (2009)

22. Shao, H: New delay-dependent stability criteria for systems with interval delay. Automatica 45, 744-749 (2009)

23. Sun, J, Liu, GP, Chen, J, Rees, D: Improved delay-range-dependent stability criteria for linear systems with time-varying delays. Automatica 46, 466-470 (2010)

24. Zhang, W, Cai, X, Han, Z: Robust stability criteria for systems with interval time-varying delay and nonlinear perturbations. J. Comput. Appl. Math. 234, 174-180 (2010)

25. Rajchakit, M, Rajchakit, G: LMI approach to robust stability and stabilization of nonlinear uncertain discrete-time systems with convex polytopic uncertainties. Adv. Differ. Equ. 2012, 106 (2012)

26. $\mathrm{Xu}, \mathrm{S}, \mathrm{Shi}, \mathrm{P}, \mathrm{Chu}, \mathrm{Y}, \mathrm{Zou}, \mathrm{Y}$ : Robust stochastic stabilization and $H_{\infty}$ control of uncertain neutral stochastic time-delay systems. J. Math. Anal. Appl. 314, 1-16 (2006) 
27. Yue, D, Won, S: Delay-dependent robust stability of stochastic systems with time delay and nonlinear uncertainties. Electron. Lett. 37, 992-993 (2001)

28. Verriest, El, Florchinger, P: Stability of stochastic systems with uncertain time delays. Syst. Control Lett. 24, 41-47 (1995)

29. Dzhalladova, IA, Bastinec, J, Diblík, J, Khusainov, DY: Estimates of exponential stability for solutions of stochastic control systems with delay. Abstr. Appl. Anal. 2011, Article ID 920412 (2011)

30. Tian, L, Liang, J, Cao, J: Robust observer for discrete-time Markovian jumping neural networks with mixed mode-dependent delays. Nonlinear Dyn. 67, 47-61 (2012)

31. Niamsup, P, Rajchakit, G: New results on robust stability and stabilization of linear discrete-time stochastic systems with convex polytopic uncertainties. J. Appl. Math. 2013, Article ID 368259 (2013). doi:10.1155/2013/368259

32. Dong, $\mathrm{H}$, Wang, $\mathrm{Z}, \mathrm{Ho}$, DWC, Gao, $\mathrm{H}$ : Robust $H_{\infty}$ filtering for Markovian jump systems with randomly occurring nonlinearities and sensor saturation: the finite-horizon case. IEEE Trans. Signal Process. 59, 3048-3057 (2011)

33. Wang, Z, Wei, G, Feng, G: Reliable $H_{\infty}$ control for discrete-time piecewise linear systems with infinite distributed delays. Automatica 45, 2991-2994 (2009)

34. Rajchakit, M, Rajchakit, G: Mean square robust stability of stochastic switched discrete-time systems with convex polytopic uncertainties. J. Inequal. Appl. 2012, 135 (2012). doi:10.1186/1029-242X-2012-135

35. Rajchakit, G: Switching design for the asymptotic stability and stabilization of nonlinear uncertain stochastic discrete-time systems. Int. J. Nonlinear Sci. Numer. Simul. 14(1), 33-44 (2013)

36. Wang, Y, Wang, Z, Liang, J: A delay fractioning approach to global synchronization of delayed complex networks with stochastic disturbances. Phys. Lett. A 372, 6066-6073 (2008)

37. Wang, Z, Wang, Y, Liu, Y: Global synchronization for discrete-time stochastic complex networks with randomly occurred nonlinearities and mixed time delays. IEEE Trans. Neural Netw. 21, 11-25 (2010)

38. Rajchakit, M, Rajchakit, G: Mean square exponential stability of stochastic switched system with interval time-varying delays. Abstr. Appl. Anal. 2012, Article ID 623014 (2012). doi:10.1155/2012/623014

39. Niamsup, P, Rajchakit, M, Rajchakit, G: Guaranteed cost control for switched recurrent neural networks with interval time-varying delay. J. Inequal. Appl. 2013, 292 (2013). doi:10.1186/1029-242X-2013-292

40. Wang, Y, Xie, L, de Souza, CE: Robust control of a class of uncertain nonlinear systems. Syst. Control Lett. 19, 139-149 (1992)

41. Boyd, S, El Ghaoui, L, Feron, E, Balakrishnan, V: Linear Matrix Inequalities in System and Control Theory. SIAM, Philadelphia (1994)

42. Uhlig, F: A recurring theorem about pairs of quadratic forms and extensions. Linear Algebra Appl. 25, 219-237 (1979)

10.1186/1029-242X-2013-499

Cite this article as: Rajchakit et al.: A constructive way to design a switching rule and switching regions to mean square exponential stability of switched stochastic systems with non-differentiable and interval time-varying delay. Journal of Inequalities and Applications 2013, 2013:499

\section{Submit your manuscript to a SpringerOpen ${ }^{\odot}$ journal and benefit from:}

- Convenient online submission

- Rigorous peer review

- Immediate publication on acceptance

- Open access: articles freely available online

- High visibility within the field

- Retaining the copyright to your article 